\title{
Design considerations for large-aperture single-mode oxide-confined vertical-cavity surface-emitting lasers
}

Cite as: Appl. Phys. Lett. 101, 071117 (2012); https://doi.org/10.1063/1.4746422

Submitted: 16 February 2012 • Accepted: 02 August 2012 • Published Online: 16 August 2012

V. P. Kalosha, N. N. Ledentsov and D. Bimberg

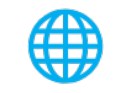

View Online

\section{ARTICLES YOU MAY BE INTERESTED IN}

Transverse-mode control in VCSELs by electrically tunable liquid crystal mode filters AIP Advances 11, 015225 (2021); https://doi.org/10.1063/5.0033685

Anomalous lasing of high-speed $850 \mathrm{~nm}$ InGaAlAs oxide-confined vertical-cavity surfaceemitting lasers with a large negative gain-to-cavity wavelength detuning Applied Physics Letters 105, 061104 (2014); https://doi.org/10.1063/1.4892885

Gain characteristics of the InGaAs strained quantum wells with GaAs, AlGaAs, and GaAsP barriers in vertical-external-cavity surface-emitting lasers

Journal of Applied Physics 105, 053103 (2009); https://doi.org/10.1063/1.3081974

耳QBLOX

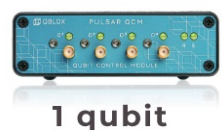

Shorten Setup Time Auto-Calibration More Qubits

Fully-integrated Quantum Control Stacks Ultrastable DC to $18.5 \mathrm{GHz}$ Synchronized $<<1$ ns Ultralow noise

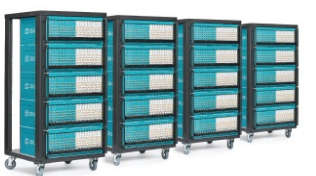

100s qubits

visit our website > 


\title{
Design considerations for large-aperture single-mode oxide-confined vertical-cavity surface-emitting lasers
}

\author{
V. P. Kalosha, ${ }^{1, a)}$ N. N. Ledentsov, ${ }^{2}$ and D. Bimberg ${ }^{1, b)}$ \\ ${ }^{1}$ Institute for Solid-State Physics and Center of NanoPhotonics, Technical University Berlin, \\ Hardenbergstr. 36, 10623 Berlin, Germany \\ ${ }^{2}$ VI Systems GmbH, Hardenbergstr. 7, 10623 Berlin, Germany
}

(Received 16 February 2012; accepted 2 August 2012; published online 16 August 2012)

\begin{abstract}
The output modal content of the oxide-confined vertical-cavity surface-emitting lasers (VCSELs) crucially depends upon the thickness of the low-index oxide aperture, its position with respect to the standing waves of the transverse-longitudinal modes and the separation from the cavity. Three-dimensional cold-cavity optical modes of typical AlGaAs/GaAs VCSELs at $850 \mathrm{~nm}$ were simulated to study these dependencies quantitatively taking into account the field diffraction and the material dispersion. Modification of one or two periods of the distributed Bragg reflector by positioning the thin oxidized aperture layers in the mode nodes allows single-mode regime to extend to the aperture diameters as large as $10 \mu \mathrm{m}$. (C) 2012 American Institute of Physics.
\end{abstract}

[http://dx.doi.org/10.1063/1.4746422]

Vertical-cavity surface-emitting lasers (VCSELs) offer low threshold, high efficiency, narrow divergence, spectral purity, high output power, integration into two-dimensional arrays, as well as mass-production capability at low cost. ${ }^{1}$ Lower and lower power consumption at ever increasing bit rates has been demonstrated, satisfying the demand of optical interconnects in data centers and in high performance computing. At the development of various VCSEL elements (such as distributed Bragg reflectors (DBRs), internal cavity, contacts, heat removal, emission output), a major break-through has been achieved using selectively oxidized aperture layers. ${ }^{2,3}$ Oxide-confined apertures provide simultaneously current and optical mode confinement due to the low conductivity and the low refractive index of the oxidized dielectric as compared to the surrounding semiconductor layers.

For both high-power industrial ${ }^{4}$ and high-speed optical communication applications of the VCSELs, ${ }^{5}$ emission of a single transverse-longitudinal mode is of crucial importance. In particular, it allows a dramatic length extension of the optical link up to very high data bit rates and energy efficiencies. ${ }^{6}$ Single-mode regime of the VCSELs is naturally offered by a small internal cavity length between the DBRs and by a small aperture size. However, for high power and low series resistance lasers, maintaining a single mode presents a challenge, since the aperture size has to be increased. A manifold of the transverse modes are inevitable at large-size oxide aperture if the oxide layer is improperly designed, making the laser output beam multi-lobed, multiwavelength and thus preventing a number of applications.

Current confinement function played by the oxide aperture allows the aperture positioning in a certain range of distances with respect to the cavity without significantly affecting the series resistance. In contrast, a specific aperture position with respect to the nodes of the vertical standing wave has a dramatic impact on mode confinement, i.e., on the mode diffraction. The smaller is the mode overlap with

\footnotetext{
${ }^{\text {a)} E l e c t r o n i c ~ m a i l: ~ v l a d i m i r . k a l o s h a @ t u-b e r l i n . d e . ~}$

b)Also at King Abdulaziz University, Jeddah 21589, Saudi Arabia.
}

the low-index aperture, the weaker is the diffraction at the aperture and the less transverse modes are possible. This effect is commonly used, however, there is a lack of studies that allow for the diffraction directly and examine the mode discrimination and selectivity quantitatively. The motivation of the present work is to address this effect on the basis of an exact solution of the wave equation and report quantitative results, illustrating the influence of the oxide aperture position and thickness on the VCSEL modal content.

For the VCSEL structures the transverse field $E(\mathbf{r})$ and the frequency $\omega$ of linearly polarized (LP) optical modes satisfy the scalar eigenvalue wave equation,

$$
-\nabla^{2} E(\mathbf{r})=(\omega / c)^{2} n^{2}(\mathbf{r}, \omega) E(\mathbf{r}),
$$

where $n(\mathbf{r}, \omega)$ is the azimuthally-symmetric distribution of the refractive index, $\nabla^{2}$ is the Laplacian in the cylindrical coordinates with the radius vector $\mathbf{r}=(r, z, \varphi)$ and $c$ is a speed of light. In Eq. (1), the field temporal dependence $\exp (i \omega t)$ is assumed and the dispersion inherent to the semiconductors manifests itself via the frequency-dependent refractive index. Since the dependence $n(\omega)$ is a nonlinear function, Eq. (1) cannot be solved directly as an eigenvalue linear system.

Following Wenzel and Wünsche, ${ }^{7}$ we use the lowestorder Taylor expansion of the dielectric constant in the vicinity of the nominal frequency $\omega_{0}$ and obtain

$$
-\left[\nabla^{2}+k_{0}^{2} n^{2}\left(\mathbf{r}, \omega_{0}\right)\right] E(\mathbf{r})=\nu n\left(\mathbf{r}, \omega_{0}\right) n_{g}\left(\mathbf{r}, \omega_{0}\right) E(\mathbf{r}) .
$$

Here $n_{g}(\mathbf{r}, \omega)=\partial[\omega n(\mathbf{r}, \omega)] / \partial \omega$ is the group index of the structure, $\nu=2 \omega_{0}\left(\omega-\omega_{0}\right) / c^{2}$ defines the frequency deviation which can be kept small by a proper choice of $\omega_{0}$ and $k_{0}=\omega_{0} / c$.

For LP modes of the azimuthally-symmetric multi-layered structures with the symmetry axis along the vertical coordinate $z$, the electric field dependence on the azimuthal coordinate is $E(\mathbf{r})=\mathcal{E}(r, z) \exp ( \pm i m \varphi)$ with $m$ as an azimuthal mode number. Then Eq. (2) is reduced to the equation,

$$
-\left[\partial_{r}^{2}+(1 / r) \partial_{r}+\partial_{z}^{2}-m^{2} / r^{2}+k_{0}^{2} n^{2}\right] \mathcal{E}=\nu n n_{g} \mathcal{E},
$$


where the arguments $(r, z)$ of the field function and $\left(r, z, \omega_{0}\right)$ of the refractive and group indices are omitted.

Now, by appropriate discretization Eq. (3) can be solved as a generalized eigenvalue linear system, where $\nu$ plays the role of the eigenvalue. As compared to Ref. 7, we do not rely on any further approximations and solve Eq. (3) exactly. Thus, within the frame of the scalar wave equation we directly take into account the field diffraction, which defines mode index-guiding by the VCSELs, as well as mode radiation from the structure, when the appropriate boundary conditions are used.

The boundary conditions for Eq. (3) should guarantee mode localization within the structure and reflection-free radiation outside at $(r, \pm z) \rightarrow \infty$. Perfectly matched layer conditions constructed using a complex coordinate stretching ${ }^{8}$ in the frequency domain have been used for that purpose. To resolve a singularity of the Laplacian at $r=0$ for $m=0$, we have used a function substitution and a radial coordinate transformation which are especially efficient for finite-difference discretization. $^{9}$ Details of the parallel numerical procedures used to solve the eigenvalue problem Eq. (3) are reported in Ref. 10.

As an example we have considered $\mathrm{Al}_{\mathrm{x}} \mathrm{Ga}_{1-x}$ As-based VCSELs designed for $\sim 850 \mathrm{~nm} .{ }^{11-13}$ These structures are consisting of 30.5 quarter-wavelength layer pairs with $\mathrm{Al}$ contents $x=1$ and 0.2 as bottom DBR, 26.5 quarter-wavelength layer pairs with $x=0.2$ and 0.9 as top DBR, one-wavelength thick inner cavity with $x=0.2$, substrate and cover with $x=0$. The active region contains three $8 \mathrm{~nm}$ thick quantum wells $(x=0)$ separated by $10 \mathrm{~nm}$ barriers $(x=0.2)$ in the middle of the inner cavity. The dispersion was found from Adachi's data. ${ }^{14} \mathrm{We}$ have assumed that the refractive and group indices $n$ and $n_{g}$ of the VCSEL depend only on the vertical distance $z$ [Fig. 1(a)], except for the high-Al layer oxidized to form the aperture. Within this layer $n=\left.n\right|_{x=0.98}$ for $r<R$ and $n=n_{\text {oxide }}$ elsewhere, where $R$ is the aperture radius and $n_{\text {oxide }}$ is the refractive index of the native oxide. ${ }^{15}$

The different solutions of the eigenstate-eigenvalue problem Eq. (3) are listed as $\mathrm{LP}_{m n}$ modes, where $m$ and $n$ count the field maxima in the angular and the radial direc-
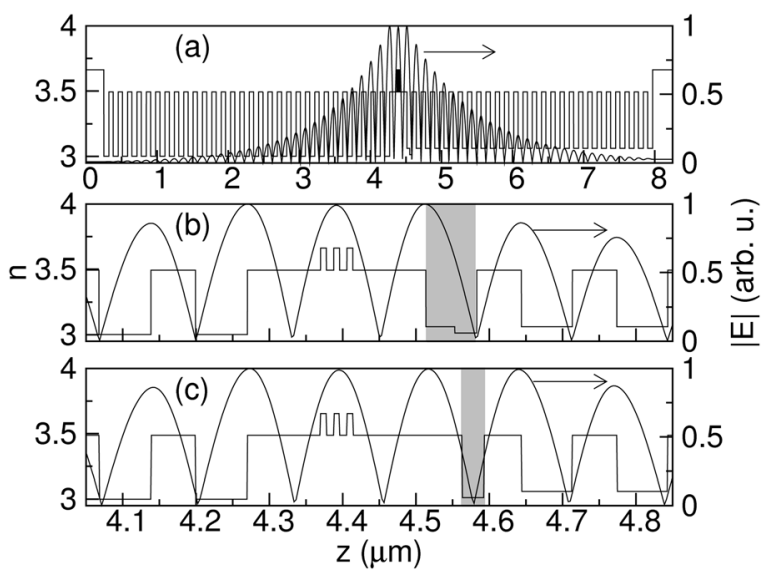

FIG. 1. Refractive index and the longitudinal mode profiles in the vertical direction for the VCSEL (see Refs. 11-13) (a), zoomed vertical profiles of the refractive index and mode field amplitude for the aperture as a $30-\mathrm{nm}$ thick oxidized aperture layer $(x=0.98)$ in the high-Al layer $(x=0.9)$ of the unchanged first period of the top DBR above the cavity (b) and for the aperture layer shifted to the mode node by modification of the first period (c). Aperture position and thickness correspond to the gray areas. tions, respectively. Due to the diffraction of the modes at the refractive index distribution of the cold-cavity VCSELs and the radiation to the outside, the mode eigenvalues $\nu$ are complex. They define the complex eigenvalue frequencies $\omega=\omega^{\prime}+i \omega^{\prime \prime}=\omega_{0}+\nu c^{2} / 2 \omega_{0}$, and hence the wavelengths $\lambda=2 \pi c / \omega^{\prime}$ and the lifetimes $\tau=1 / \omega^{\prime \prime}$ or $Q$-factors $Q=\omega^{\prime} / 2 \omega^{\prime \prime}$ of the cavity modes.

There exists an obvious correspondence between the mode lifetime and mode gain threshold, and the longer the lifetime is, the lower is the threshold. Thus the mode field diffraction and radiation in the radial and the vertical directions as well as the back reflection and transmission at the DBRs are self-consistently evaluated. To characterize the discrimination of the cold-cavity laser modes further, the mode confinement factors $\Gamma$ are also considered. They are calculated as portions of the mode powers proportional to $|\mathcal{E}|^{2}$ in the QWs and within the aperture $r<R$. The modes with larger both lifetime and confinement factor are favored for lasing as compared to the modes with smaller $\tau$ and $\Gamma$.

Single longitudinal mode of the VCSEL is shown in Fig. 1(a) obtained by the solution of Eq. (2) in 1-D, i.e., without taking into account the radial coordinate dependence and the aperture role. This mode has a wavelength of $850.8 \mathrm{~nm}$, a confinement factor of $3.5 \%$ and a lifetime of 16.8 ps defined mostly by the mode radiation into the cover layer above the top DBR.

Oxidation allows precise control of the aperture radius, thickness and shape, when the oxidized layer has adjacent layers with considerably different $\mathrm{Al}$ contents. ${ }^{16}$ We consider two cases for the aperture location in the top DBR period nearest to the cavity. First, the oxidized layer $(x=0.98)$ is located within the high-Al content layer $(x=0.9)$ of the unchanged DBR period close to the field node. Small Alcontent difference of the layers usually results in the oxidation of the entire $\lambda / 4$ high-Al content layer with a tapered shape of the aperture. ${ }^{17}$ However, this short tapered edge can be neglected for the large-size aperture and we model the aperture by a quarter-wavelength thick layer of the native oxide (Fig. 1(b)). Second, the high-Al content layer is intentionally made thinner, shifted precisely to the node of the longitudinal mode by a corresponding modification of the internal cavity and the closest Bragg period and substituted by the oxidized layer (Fig. 1(c)).

In the first case, a 5- $\mu \mathrm{m}$ radius aperture provides strong lateral confinement of a large number of the transverselongitudinal LP modes (more than 40). Their shapes for $m \leq 4$ and $n \leq 3$ are shown in the plane $(r, z)$ in Fig. 2. Although the aperture is thin as compared to the mode extension, it intersects the field anti-node [Fig. 1(b)]. This explains strong mode diffraction and so essential role of the aperture in confinement of many modes.

Strong diffraction by the aperture is clearly illustrated in Fig. 3 for this case, where we show the near-field of the modes at the edge of the cover layer. All maxima of the fields are normalized to unity, to visualize diffracted parts of the output outside the aperture region (limited by the black circles). The output of the modes is also illustrated by their far-fields (Fig. 4). They are shown in the transverse angular coordinates $\left(\theta_{x}, \theta_{y}\right)$ related to the transverse wavenumbers as $k_{x, y}=k_{0} \sin \theta_{x, y}$. The higher-order mode far-fields have 


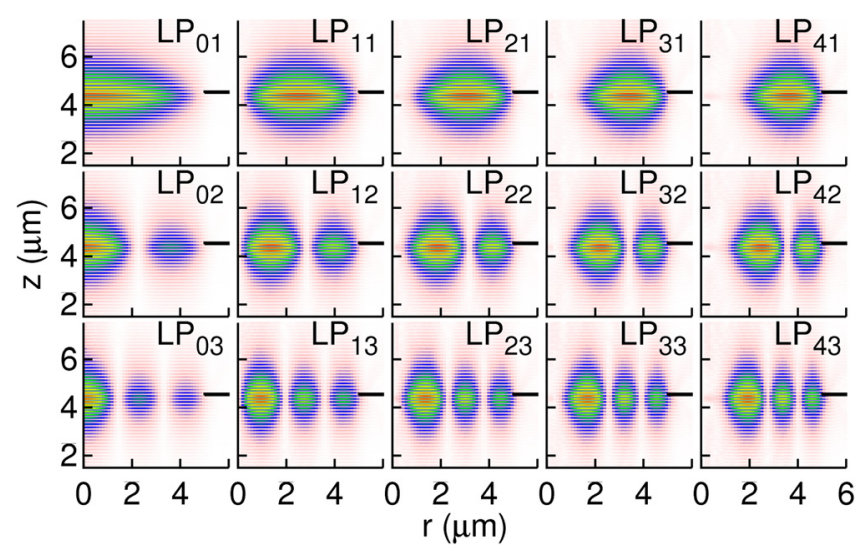

FIG. 2. Transverse-longitudinal $\mathrm{LP}_{m n}$ modes of the VCSEL with the aperture of $5 \mu \mathrm{m}$ radius and thickness of the quarter-wavelength high-Al content layer [Fig. 1(b)] for the azimuthal number $m=0,1,2,3$, and 4 (columns from left to right) and the radial number $n=1,2$, and 3 (rows from top to bottom). Field amplitude $|\mathcal{E}|$ is shown in map view versus the radial and the vertical coordinates. The aperture edges are shown by the black segments with the corresponding thickness.

multi-lobed patterns, typical for azimuthally-symmetric structures and corresponding to the mode orders $m$ and $n$.

The lifetimes and the confinement factors of the modes for the first case of the aperture in the unchanged DBR versus the mode wavelength are presented in Fig. 5 (data set (1)). The actual total number of modes for that case is much larger, since we restricted ourselves by $m, n \leq 5$. The modes demonstrate a wavelength blue shift and the decreasing lifetime with increasing the mode azimuthal and radial orders. Smaller values of the lifetime obtained for 3-D modes, than those obtained in 1-D case (Fig. 1), characterize the contribution of the diffraction and radiation into the transverse directions.

As one can see from the data sets (2) and (3) in Fig. 5, the effect of thinning the rather large aperture of $10 \mu \mathrm{m}$ diameter and shifting it to the node position is enormous. The number of the confined modes is drastically decreased with decreasing the aperture thickness. There is no selection by the confinement factor in the case of thick aperture in the unchanged DBR, whereas selection occurs for the thin aperture in the field nodes. A red shift of the mode wavelengths in the data sets from (1) to (3) (Fig. 5) is obviously associ-

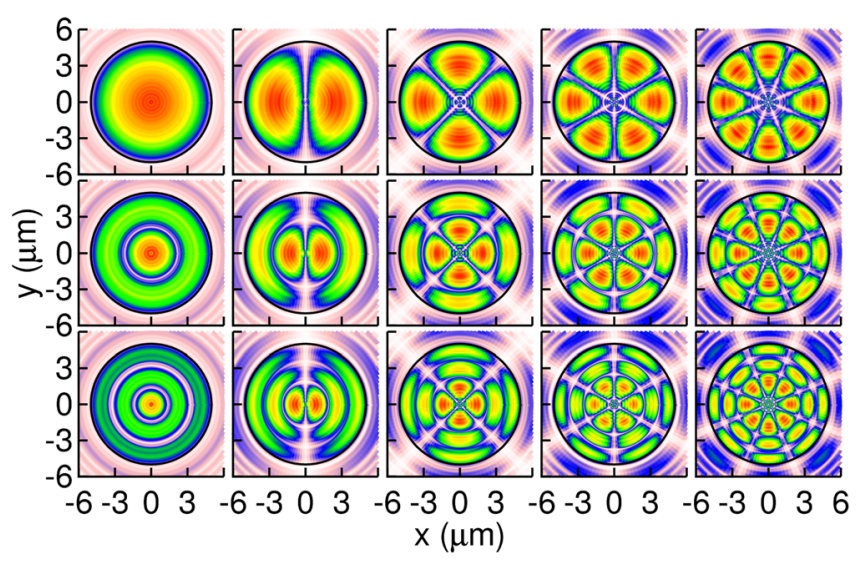

FIG. 3. Output near-field of the $\mathrm{LP}_{m n}$ modes as in Fig. 2. Real part of the output field is shown versus the transverse Cartesian coordinates. The $5 \mu \mathrm{m}$ radius aperture edge is shown by the black circle.

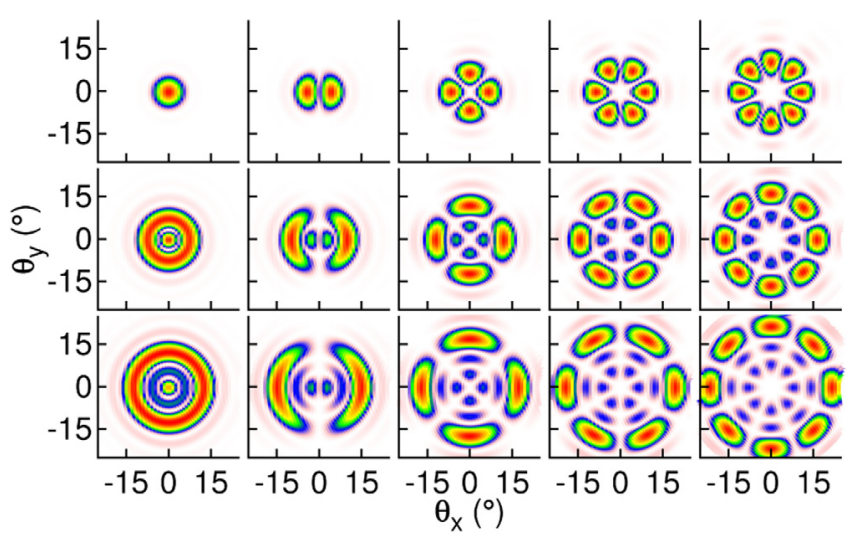

FIG. 4. Output far-field of $\mathrm{LP}_{m n}$ modes for the same nomenclature as in Figs. 2 and 3. Field intensity is shown versus transverse angular coordinates.

ated with an increase of mean refractive index of the structure with the aperture thinning. Then, similar to a FabryPerot resonator, a conditional optical path of the modes remains resonant at lower frequencies.

To obtain true single transverse-longitudinal mode operation by weakening the field diffraction at the aperture, we have considered a similar modification of the second and the third period of the top DBR [Fig. 6(a)]. In contrast to the previous case (Fig. 1), this modification does not change the inner cavity which remains to be one-wavelength thick. For a given aperture thickness a gradual decrease of the number of the confined modes occurs by moving the aperture away from the cavity. For a 30-nm thick aperture in the mode nodes, three $\mathrm{LP}_{m n}$ modes are confined with $m n=01,11,21$ when the aperture is positioned in the first period (data set (3) in Fig. 5), two modes with $m n=01,11$ when the aperture is in the second period, and only one $\mathrm{LP}_{01}$ mode when the aperture is in the third period. Simultaneously, the mode lifetime is also decreased. In the true single-mode regime, the mode has a wavelength of $854.7 \mathrm{~nm}$, a confinement factor of $2.3 \%$ and a lifetime of $13.2 \mathrm{ps}$.

The transverse-longitudinal pattern and the transverse output near-field of the single mode confined by the oxide aperture with a large radius of $5 \mu \mathrm{m}$ are presented in

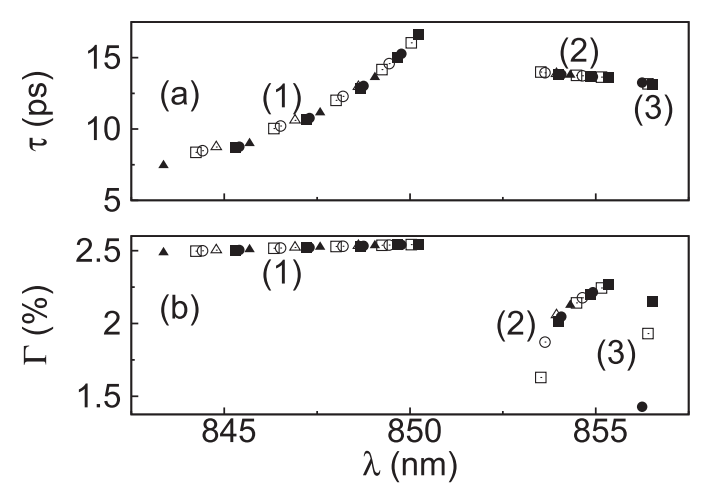

FIG. 5. Lifetime (a) and confinement factor (b) of the VCSEL modes (1) for the aperture formed by high-Al content layer in the unchanged top DBR [Fig. $1(\mathrm{~b})]$ and (2) for the aperture formed by high-Al content layer of the thickness $50 \mathrm{~nm}$ and (3) $30 \mathrm{~nm}$ [Fig. 1(c)] shifted to the mode node. Different point styles correspond to the different azimuthal modes: $m=0(\boldsymbol{\square}), 1(\square), 2(\mathbf{O})$, $3(\bigcirc), 4(\mathbf{\Delta}), 5(\triangle)$; the same points in the given data set correspond to the different mode radial numbers. Only a part of all the modes is shown for (1). 

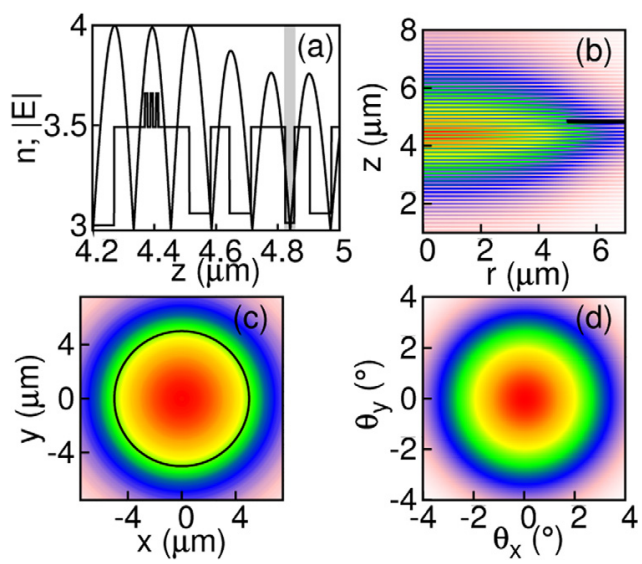

FIG. 6. (a) Refractive index and longitudinal mode profile versus the vertical coordinate in the vicinity of the VCSEL cavity and 30-nm thick oxidized layer (gray area) shifted to the mode node in the third DBR period. (b) Transverse-longitudinal pattern of $\mathrm{LP}_{01}$ mode. (c) Near- and (d) far-field transverse pattern of $\mathrm{LP}_{01}$ mode. Aperture edges are shown by black segment (b) and circle (c).

Figs. 6(b) and 6(c), respectively. They illustrate the weak diffraction of the mode and field spreading well beyond the aperture for this case of the aperture thickness and location. Correspondingly, the mode has a narrow output far-field with as small full width at half-maximum as $3.7^{\circ}$ (Fig. 6(c)).

Finally, we note that the theory of the mode behavior in oxide-confined VCSELs should consider a complicated selfconsistent interrelation between the optical field, the gain, the current transport and the thermal flow (see, e.g., Refs. 18-20). Only such a theory can predict ultimate laser characteristics like output power, spectrum, output near- and farfield profiles versus applied voltage. Nevertheless, optical simulations alone deliver already important insight towards a proper VCSEL design. Mode selectivity remains the same as shown in Fig. 5, if an inhomogeneous optical gain in the active layers and within the aperture is taken into consideration. The same refers to the wavelength shift for thin apertures.

In conclusion, we have studied quantitatively the role of the position and the thickness of the apertures on the mode discrimination and selectivity in the oxide-confined VCSELs. Simulations of 3-D cold-cavity transverse-longitudinal LP modes of typical AlGaAs/GaAs VCSELs emitting at $850 \mathrm{~nm}$ were performed, taking into account the field diffraction and radiation, as well as the material dispersion of the layers com- prising the VCSEL. The discrimination of the modes was characterized by the mode lifetimes and the confinement factors. We have shown that the modification of the structure within the region of only a few layer pairs of the top DBR by thinning high-Al content aperture layers and by shifting them to the mode nodes provides single-mode operation for large apertures.

V.P.K. and D.B. acknowledge support of the DFG within SFB 787 "Semiconductor Nanophotonics" and ProFit project Bright. N.N.L. acknowledges support of the Sepianet project within PIANO+ program.

${ }^{1}$ K. Iga, Jpn. J. Appl. Phys. 47, 1 (2008).

${ }^{2}$ A. R. Sugg, E. I. Chen, T. A. Richard, N. Holonyak, and K. C. Hsieh, Appl. Phys. Lett. 62, 1259 (1993).

${ }^{3}$ D. L. Huffaker, D. G. Deppe, K. Kumar, and T. J. Rogers, Appl. Phys. Lett. 65, 97 (1994).

${ }^{4}$ J.-F. Seurin, G. Xu, Q. Wang, B. Guo, R. Van Leeuwen, A. Miglo, P. Pradhan, J. D. Wynn, V. Khalfin, and C. Ghosh, Proc. SPIE 7615, 76150F (2010).

${ }^{5}$ S. A. Blochin, J. A. Lott, A. Mutig, G. Fiol, N. N. Ledentsov, M. V. Maximov, A. M. Nadtochiy, V. A. Shchukin, and D. Bimberg, Electron. Lett. 45, 501 (2009).

${ }^{6}$ P. Moser, J. A. Lott, P. Wolf, G. Larisch, A. Payusov, N. N. Ledentsov, W. Hofmann, and D. Bimberg, IEEE Photonics Technol. Lett. 24, 19 (2012).

${ }^{7}$ H. Wenzel and H.-J. Wünsche, IEEE J. Quantum Electron. 33, 1156 (1997).

${ }^{8}$ F. L. Teixeira and W. C. Chew, IEEE Mircow. Guid. Wave Lett. 7, 285 (1997).

${ }^{9}$ H. Kono, A. Kita, Y. Ohtsuki, and Y. Fujimura, J. Comput. Phys. 130, 148 (1997).

${ }^{10}$ V. P. Kalosha, K. Posilovic, and D. Bimberg, IEEE J. Quantum Electron. 48, 123 (2012).

${ }^{11}$ R. Jäger, M. Grabherr, C. Jung, R. Michalzik, G. Reiner, B. Weigl, and K. J. Ebeling, Electron. Lett. 33, 330 (1997).

${ }^{12}$ C. Jung, R. Jäger, M. Grabherr, P. Schnitzer, R. Michalzik, B. Weigl, S. Müller, and K. J. Ebeling, Electron. Lett. 33, 1790 (1997).

${ }^{13}$ C. Degen, I. Fischer, and W. Elsäßer, Opt. Express 5, 38 (1999).

${ }^{14}$ S. Adachi, J. Appl. Phys. 58, R1 (1985).

${ }^{15}$ K. J. Knopp, R. P. Mirin, D. H. Christensen, K. A. Bertness, A. Roshko, and R. A. Synowicki, Appl. Phys. Lett. 73, 3512 (1998).

${ }^{16}$ P. Moser, W. Hofmann, P. Wolf, J. A. Lott, G. Larisch, A. Payusov, N. N. Ledentsov, and D. Bimberg, Appl. Phys. Lett. 98, 231106 (2011).

${ }^{17}$ Y.-C. Chang and L. A. Coldren, IEEE J. Sel. Topics Quantum Electron. 15, 704 (2009).

${ }^{18}$ J. Piprek, H. Wenzel, H.-J. Wünsche, D. Braun, and F. Henneberger, Proc. SPIE 2399, 605 (1995).

${ }^{19}$ T. Rössler, R. A. Indik, G. K. Harkness, J. V. Moloney, and C. Z. Ning, Phys. Rev. A 58, 3279 (1998).

${ }^{20}$ M. Streiff, A. Witzig, M. Pfeiffer, P. Royo, and W. A. Fichtner, IEEE J. Sel. Top. Quantum Electron. 9, 879 (2003). 